Pacific Journal of Mathematic 


\title{
ABSOLUTE CONTINUITY FOR ABSTRACT WIENER SPACES
}

\author{
T. E. DunCan
}

\begin{abstract}
Certain smooth homeomorphisms on an abstract Wiener space are shown to induce a measure that is absolutely continuous with respect to an abstract Wiener measure which is the measure determined on a Banach space from the canonical normal distribution on a Hilbert space by the completion of the Hilbert space with respect to a measurable seminorm. The notion of an abstract Wiener space is an abstraction of one technique to show the countable additivity for Wiener measure, the measure for Brownian motion. The generalizations of absolute continuity obtained here reduce exactly to the well known results for absolute continuity for Gaussian measures.
\end{abstract}

Specifically, it is shown that if $T$ is a $C^{1}$ diffeomorphism of the Banach space such that $T=I+\psi$ where $I$ is the identity and $\psi$ takes values in the Hilbert space associated with the abstract Wiener space and is differentiable, then the measure induced on the Banach space by the transformation, $T$, is absolutely continuous with respect to the abstract Wiener measure.

2. Preliminaries. Let $(i, H, B)$ be an abstract Wiener space where $H$ is a separable Hilbert space, $B$ is a separable Banach space obtained from $H$ by completing $H$ with respect to the measurable seminorm, $|\cdot|$, and $i: H \rightarrow B$ is the continuous canonical injection. $\mathscr{F}$ will denote the Borel $\sigma$-algebra on $B$. For fundamental notions about these measures the reader is referred to [1, 3].

Let $\nu$ be the canonical normal distribution on $H$ with variance parameter $t=1$ and let $\mu$ be the measure induced on $B$ as the projective limit of the family of cylinder set measures on $B$ obtained from $\nu$ and the continuous canonical injection, $i$. Assuming the variance parameter is 1 is inessential for the results for absolute continuity; any variance parameter $t \in(0, \infty)$ could be used with trivial changes in the subsequent results for absolute continuity. For some previous related results on absolute continuity, the reader is referred to [5].

The following definition provides some notation that is used subsequently.

Definition. $\mathscr{P}=\{P: P$ is a finite dimensional projection on $H$ with $\left.P H \subset j B^{*}\right\}$ where $B^{*}$ is the topological dual of $B$ and $j=i^{*}$. 
In order to establish absolute continuity a few preliminary results have to be obtained. The first result is contained in a result of Gross (Cor. 3 [3]).

Lemma 1. Let $(i, H, B)$ be an abstract Wiener space and let $\left(P_{n}\right)_{n \in N}$ be a sequence of finite dimensional projections on $H$ such that $P_{n} \rightarrow I$ where $I$ is the identity on $H$. If $f: B \rightarrow \boldsymbol{R}$ is continuous then $f_{n}^{\sim} \rightarrow f$ in probability where $f_{n}^{\sim}$ is the random variable on $B$ induced by the cylinder set function $f \circ i \circ P_{n}$ on $H$.

For absolute continuity for Gaussian measures on a Banach space and linear transformations on the Banach space, certain HilbertSchmidt operators play a fundamental role, [6]. Likewise, for smooth homeomorphisms on a Banach space and questions of absolute continuity Hilbert-Schmidt operators again arise naturally as one would expect from a local analysis.

The proof of the following lemma which replaced a less succinct proof by the author was supplied by the referee.

Lemma 2. Let $A: B \rightarrow H$ be a continuous linear operator. Then $A \mid H$ is a Hilbert-Schmidt operator.

Proof. If $S: B \rightarrow B^{*}$ is a continuous linear operator then $S \mid H: H \rightarrow H$ is a trace class operator. (This nice theorem is due to V. Goodman.)

Now to prove the lemma consider $(A \mid H)^{*} \circ A: B \rightarrow H \rightarrow H$. Observe that if $x \in B$ then for all $h \in H$

$$
\begin{aligned}
\frac{\left|\left\langle(A \mid H)^{*} \circ A x, h\right\rangle_{H}\right|}{|h|} & =\frac{\left|\langle A x,(A \mid H) h\rangle_{H}\right|}{|h|} \\
& \leqq \frac{|A x|_{H}|A h|_{H}}{|h|} \\
& \leqq K \frac{|A x|_{H}|h|}{|h|} \\
& =K|A x|_{H}
\end{aligned}
$$

where $K$ is the operator norm of $A: B \rightarrow H$. Therefore, $(A \mid H)^{*} \circ A x \in$ $B^{*}$ and $(A \mid H)^{*} \circ A(B) \subset B^{*}$. Now an application of the closed graph theorem shows that $(A \mid H)^{*} \circ A: B \rightarrow B^{*}$ is a continuous linear operator. Hence $(A \mid H)^{*} \circ(A \mid H): H \rightarrow H$ is a trace class operator and $A \mid H: H \rightarrow H$ is a Hilbert-Schmidt operator.

Uniform integrability plays a fundamental role in questions of absolute continuity, because of its equivalence to weak compactness 
in $L^{1}$. The following result gives some sufficient conditions for uniform integrability of a family of Radon-Nikodym derivatives.

Lemma 3. Let $T: B \rightarrow B$ be a $C^{1}$ diffeomorphism where $T=I+$ $\psi, I$ is the identity on $B$ and $\psi: B \rightarrow H$. Assume that there is an $\alpha>1$ such that $(I+\alpha \psi) \mid H$ is a diffeomorphism on $H$. Assume that $|\psi|_{H}$ and $\left.\left|\psi^{\prime}\right| H\right|_{H S}$ are uniformly bounded on $B$ where $|\cdot|_{H}$ is the norm on $H$ induced by the inner product and $|\cdot|_{H S}$ is the HilbertSchmidt norm. Furthermore, let $\left(\lambda_{n}\right)_{n \in N}$ be the eigenvalues for $\psi^{\prime} \mid H$ and assume that the Fredholm-Carleman determinants

$$
\prod_{n=1}^{\infty}\left(1+\lambda_{n}\right) e^{\lambda_{n}}
$$

and

$$
\prod_{n=1}^{\infty}\left(1+\alpha \lambda_{n}\right) e^{\alpha \lambda_{n}}
$$

are bounded above and away from 0 uniformly for $x \in B$.

Let $T_{\widetilde{P}}=\widetilde{P} I+\widetilde{P} \psi \widetilde{P}$ be the diffeomorphism on $\widetilde{P} B$ where $\widetilde{P}$ is the continuous finite dimensional projection on $B$ induced from $P \in \mathscr{P}$ and let $f_{\widetilde{P}}$ be the function on $B$ induced by the Radon-Nikodym derivative for the transformation of measures on $\widetilde{P} B$ detemined by $T_{\widetilde{P}}$.

Then the family of Radon-Nikodym derivatives

$$
\left(f_{\tilde{P}}\right)_{P \in \mathscr{O}}
$$

is uniformly integrable.

Proof. Let $T_{P}$ be the restriction of $T_{\widetilde{P}}$ to $P H$. The canonical normal distribution restricted to $P H$ is a measure and is transformed by absolute continuity of measures by the transformation, $T_{P}$, because $P I+P \psi P$ is a $C^{1}$ diffeomorphism on $P H$. The identity operator on $H$ or $B$ will be denoted by the same symbol, $I$, because the appropriate space will be clear from the context. Let $d \nu_{T_{P}} / d \nu_{P}$ be the Radon-Nikodym derivative corresponding to this diffeomorphism on $P H$. Writing $d \nu_{T_{P}} / d \nu_{P}$ as a cylinder set function on $H$ it follows that

$$
\frac{d \nu_{T_{P}}}{d \nu_{P}}(x)=\operatorname{det}\left|I+P A_{P x} P\right| \exp \left[(P \psi P x, x)-\frac{1}{2}|P \psi P x|_{H}^{2}\right]
$$

where $A=\psi^{\prime} \mid H$. Since this Radon-Nikodym derivative is continuous, by Gross (Cor. 3 [3]), it follows that

$$
f_{\widetilde{P}}(x)=\operatorname{det}\left|I+P A_{\widetilde{P} x} P\right| \exp \left[(P \dot{\psi} \widetilde{P} x, x)-\frac{1}{2}\left|P \psi \widetilde{P}_{x}\right|_{H}^{2}\right]
$$


where $f_{\widetilde{P}}$ is the Radon-Nikodym derivative, $d \mu_{T_{\tilde{P}}} / d \mu_{\tilde{P}}$, considered as a function on $B$.

To verify the uniform integrability of the family, $\left(f_{\tilde{P}}\right)_{P \in \mathscr{F}}$, it suffices to show that there is a $\beta>1$ such that

$$
\sup _{P \in \mathscr{P}} \int\left|f_{\tilde{P}}\right|^{\beta} d \mu<\infty \text {. }
$$

Let $\beta=\sqrt{\alpha}>1$ where $\alpha$ is given in the statement of the lemma. Fix $P \in \mathscr{P}$. Define

$$
\begin{gathered}
g(x)=\left[\operatorname{det}\left|I+\beta^{2} P A_{\widetilde{P} x} P\right| e^{\beta^{2} \operatorname{tr} P \widetilde{P}_{\widetilde{P}} P}\right]^{1 / \beta} \\
\exp \left[\beta(P \psi \widetilde{P} x, x)-\beta \operatorname{tr} P A_{\widetilde{P} x} P-\frac{\beta^{3}}{2}|P \psi \widetilde{P} x|_{H}^{2}\right] \\
h(x)=\frac{\left[\operatorname{det}\left|I+P A_{\widetilde{P} x} P\right| e^{\operatorname{tr} P A_{\widetilde{P} x} P}\right]^{\beta}}{\left[\operatorname{det}\left|I+\beta^{2} P A_{\widetilde{P} x} P\right| e^{\beta^{2} \operatorname{tr} P \widetilde{P}_{P} P}\right]^{1 / \beta}} \exp \left[\frac{\beta^{3}-\beta}{2}\left|P \psi \widetilde{P}_{x}\right|_{H}^{2}\right] .
\end{gathered}
$$

Clearly

$$
\left|f_{\widetilde{P}}\right|^{\beta}=g h .
$$

Applying Hölder's inequality to $g h$ yields

$$
E g h \leqq\left[E g^{\beta}\right]^{1 / \beta}\left[E h^{\beta /(\beta-1)}\right]^{(\beta-1) / \beta} .
$$

Since

$$
g^{\beta}=\frac{d \mu_{I+\alpha P}, \widetilde{P}}{d \mu_{\widetilde{P}}}
$$

it follows that

$$
E g^{\beta}=1 \text {. }
$$

By assumption, $h$ is bounded independent of $P \in \mathscr{P}$ so the family of Radon-Nikodym derivatives, $\left(f_{\widetilde{P}}\right)_{P \in \mathscr{P}}$, is uniformly integrable.

Using the uniform integrability results from Lemma 3, a measure can be obtained that corresponds to the transformation, $T$, as the following result describes.

Lemma 4. Let $T: B \rightarrow B$ be a $C^{1}$ diffeomorphism where $T=I+\psi$, $I$ is the identity on $B$ and $\psi: B \rightarrow H$. Assume that $T$ satisfies the hypotheses given in Lemma 3. Let $\left(P_{n}\right)_{n \in N}$ be an increasing sequence of elements of $\mathscr{P}$ such that $P_{n} \rightarrow I$ in $H$.

Then there is a subsequence of the Radon-Nikodym derivatives, $\left(f_{\widetilde{P}_{n}}\right)_{n \in N}$, that converge to a $g \in L^{1}$ in the topology $\sigma\left(L^{1}, L^{\infty}\right)$. Let $\mu_{T}$ be the measure induced on $(B, \mathscr{F})$ by the identification $d \mu_{T}=g d \mu$. The transformation $I-\psi: B \rightarrow B$ on $\left(B, \mathscr{F}, \mu_{T}\right)$ induces an abstract 
Wiener measure on $B$ with Hilbert space $H$, i.e.; for each $l \in H^{*}$, $(l, I-\psi)$ is a Gaussian random variable on $\left(B, \mathscr{F}, \mu_{r}\right)$ with zero mean and variance $|l|_{I I}^{2}$.

Proof. Since the family, $\left(f_{\widetilde{P}_{n}}\right)_{n \in N}$, is uniformly integrable there is a subsequence that converges to a function $g \in L^{1}$ in the topology $\sigma\left(L^{1}, L^{\infty}\right)$ by the weak compactness criterion of Dunford-Pettis (p. 294 [2]).

Let $f: B \rightarrow \boldsymbol{R}$ be a bounded continuous function and let $K$ be a constant that bounds $f$. By reducing the original sequence of RadonNikodym derivatives to a suitable subsequence it can be assumed that the whole sequence $\left(f_{\widetilde{P}_{n}}\right)_{n \in N}$ of Radon-Nikodym derivatives converges in $\sigma\left(L^{1}, L^{\infty}\right)$.

Since $P_{n} \rightarrow I$ and $f$ is continuous $f \circ \widetilde{P}_{n} \rightarrow f$ in probability by Lemma 1. By going to a subsequence if necessary, it can be assumed that $f \circ \widetilde{P}_{n} \rightarrow f$ a.e. Again for notational convenience, it is assumed that the whole sequence converges a.e.

By uniform integrability given $\varepsilon>0$ there is a $\delta>0$ such that if $A$ is a measurable set with $\mu A<\delta$ then

$$
\int_{A} f_{\widetilde{P}_{n}} d \mu<\varepsilon \quad \forall n \in N
$$

By Egorov's theorem, for $\delta>0$ given above there is a measurable set $A$ with $\mu A<\delta$ such that $f \circ \widetilde{P}_{n} \rightarrow f$ uniformly on $A^{c}$. For $\varepsilon>0$ there is an integer $M$ such that if $m \geqq M$

$$
\sup _{x \in A^{c}}\left|f \widetilde{P}_{m}(x)-f(x)\right|<\varepsilon .
$$

Likewise for $\varepsilon>0$ by $\sigma\left(L^{1}, L^{\infty}\right)$ convergence there is an integer $N$ such that for $n \geqq N$

$$
\left|\int f f_{\widetilde{P}_{n}}-\int f g\right|<\varepsilon .
$$

Let $n \geqq N \vee M$, then

$$
\begin{aligned}
& \int\left|f_{n}-f\right| f_{\widetilde{P}_{n}} d \mu \\
& \quad=\int_{A^{c}}\left|f_{n}-f\right| f_{\widetilde{P}_{n}} d \mu+\int_{A}\left|f_{n}-f\right| f_{\widetilde{P}_{n}} d \mu \leqq \varepsilon+2 K \varepsilon
\end{aligned}
$$

and

$$
\left|\int f_{n} f_{\widetilde{P}_{n}}-\int f g\right| \leqq 2 \varepsilon(1+K)
$$

where $f_{n}=f \circ \widetilde{P}_{n}$. 
It suffices for the verification of an abstract Wiener space to consider $l \in B^{*}$ such that $\widetilde{P}_{l} \subset \widetilde{P}_{n}$ for some $n$ where $\widetilde{P}_{l}$ is the one dimensional projection determined by $l$. Given $\varepsilon>0$ by the above results there is an $N$ such that if $n \geqq N$

$$
\left|\int f_{n} f_{\widetilde{P}_{n}}-\int f g\right|<\varepsilon
$$

where $f=e^{i t(l, I-\psi)}$ and $t \in \boldsymbol{R}$.

Furthermore, there is a compact set $K \subset B$ such that $P(K)>$ $1-\delta$ where $\delta>0$ is as above. For $l \in B^{*}$ given above there is an $M$ such that for $n \geqq M \widetilde{P}_{l} \subset \widetilde{P}_{n}$ and for $x \in K$

$$
\left|e^{-i t\left(l, P_{n} \psi\right)}-e^{-i t(l, \psi)}\right|<\varepsilon
$$

Thus, for $k \geqq M \vee N$

$$
\left.\mid \int e^{i t l l, I-P_{k} \psi \tilde{P}_{k}}\right) f_{\widetilde{P}_{k}}-\int f g \mid<4 \varepsilon .
$$

Therefore, by the transformation of measures results for finite dimensional spaces and the convergence of the characteristic functions, $(l, I-\psi)$ is a zero mean Gaussian random variable with variance $|l|_{H}^{2}$ on the probability space $\left(B, \mathscr{F}, \mu_{T}\right)$.

3. Main result. From the results for absolute continuity of Gaussian measures on an abstract Wiener space the following result for absolute continuity for a smooth homeomorphism on $B$ is the best that can be expected.

Theorem. Let $T: B \rightarrow B$ be a $C^{1}$ diffeomorphism such that $T=$ $I+\psi$ where $I$ is the identity on $B$ and $\psi: B \rightarrow H$ is differentiable. Then the measure, $\mu_{T}$, induced on $B$ by the transformation, $T$, on the abstract Wiener probability space, $(B, \mathscr{F}, \mu)$, is absolutely continuous with respect to $\mu$.

Proof. By examination of the proof of Lemma 3, there is also a local version of the result that is applicable here. The local version follows because if $U$ is an open subset of $B$ such that when $T^{-1}$ is restricted to $U$ the hypotheses of the lemma are satisfied for $T$ then the Radon-Nikodym derivatives can be computed by the change of variables formula for finite dimensional spaces and because the integrations are on $U$ the function $h$ in the proof of Lemma 3 is still uniformly bounded.

For each $x \in B$ there is an open sphere with center $x, S(x)$, such that when $T^{-1}$ is restricted to $S(x)$ the hypotheses of Lemma 3 are 
satisfied for $T$. This verification is made as follows. Recall that $\psi$ is continuously differentiable and $\psi^{\prime} \mid H$ is continuous as a map from $B$ into Hilbert-Schmidt operators. Furthermore, since $\psi^{\prime} \mid H$ is a Hilbert-Schmidt operator the Fredholm-Carleman determinant is well defined by a simple comparison test for infinite series. For $x_{0} \in B$ fixed there is an $\alpha>1$ such that $\left(I+\alpha \psi^{\prime}\left(x_{0}\right) \mid H\right)$ is invertible and such that there is a neighborhood of $x_{0}$ where this is also true.

Let $f$ be a bounded continuous function with support in $S\left(x_{0}\right)$. For each $m \in N f \circ \widetilde{P}_{m} \circ \widetilde{P}_{n} \circ T \circ \widetilde{P}_{n} \rightarrow f \circ \widetilde{P}_{m} \circ T$ in probability on $(B, \mathscr{F}$, $\mu)$ as $n \rightarrow \infty$ and $f \circ \widetilde{P}_{m} \rightarrow f$ in probability on $(B, \mathscr{F}, \mu)$ as $m \rightarrow \infty$ where $\left(P_{m}\right)$ is an increasing sequence of elements of $\mathscr{P}$ such that $P_{m} \rightarrow I$. By the bounded convergence theorem

$$
\lim _{n \rightarrow \infty} \int f \circ \widetilde{P}_{m} \circ \widetilde{P}_{n} \circ T \circ \widetilde{P}_{n} d \mu=\int f \circ \widetilde{P}_{m} \circ T d \mu
$$

in the $L^{1}(\mu)$ topology.

For $n \geqq m$

$$
\int f \circ P_{m} \circ P_{n} \circ T \circ P_{n} d \mu=\int_{\widetilde{P}_{n} B} f_{m} \circ T_{n} d \mu_{\tilde{P}_{n}}
$$

where $f_{m}$ is the continuous function induced on $\widetilde{P}_{n} B$ by $f \circ \widetilde{P}_{m}, T_{n}$ is the homeomorphism on $P_{n} B$ induced from $\widetilde{P}_{n} \circ T \circ \widetilde{P}_{n}$ and $\mu_{\tilde{P}_{n}}$ is the measure induced on $\widetilde{P}_{n} B$ by restricting $\mu$ to $\widetilde{P}_{n} B$.

By the results for the transformations of measures on finite dimensional spaces that have been noted in the proof of Lemma 3

$$
\begin{aligned}
\int f_{m} \circ T_{n} d \mu_{\widetilde{P}_{n}} & =\int f_{m} d \mu_{T_{n}} \\
& =\int f_{m} \frac{d \mu_{T_{n}}}{d \mu_{\widetilde{P}_{n}}} d \mu_{\widetilde{P}_{n}}
\end{aligned}
$$

where $\mu_{T_{n}}$ is the measure on $\widetilde{P}_{n} B$ induced by the transformation $T_{n}$ on $\left(\widetilde{P}_{n} B, \mu_{\widetilde{P}_{n}}\right)$. Considering $d \mu_{T_{n}} / d \mu_{\widetilde{P}_{n}}$ as a function on $B, f_{\widetilde{P}_{n}}$, as noted in the proof of Lemma 3 and recalling how $f_{m}$ was obtained the following equation is established

$$
\int f_{m} \circ T_{n} d \mu_{\widetilde{P}_{n}}=\int f \circ P_{m} f_{\widetilde{P}_{n}} d \mu .
$$

Assuming for notational convenience that the whole sequence of Radon-Nikodym derivatives, $\left(f_{\widetilde{P}_{n}}\right)_{n \in N}$, converges in $\sigma\left(L^{1}, L^{\infty}\right)$, then

$$
\lim _{n \rightarrow \infty} \int f \circ \widetilde{P}_{m} f_{\widetilde{P}_{n}} d \mu=\int f \circ \widetilde{P}_{m} g d \mu .
$$

By the dominated convergence theorem 


$$
\lim _{m \rightarrow \infty} \int f \circ \widetilde{P}_{m} g d \mu=\int f g d \mu .
$$

Since $T$ is a measurable mapping on $(B, \mathscr{F})$ and using the bounded convergence theorem it follows that

$$
\lim _{m \rightarrow \infty} \int f \circ \widetilde{P}_{m} \circ T d \mu=\int f \circ T d \mu=\int f d \mu_{T} .
$$

By (2) and (3) and the dominated convergence theorem, we have

$$
\lim _{m \rightarrow \infty} \int f \circ \widetilde{P}_{m} \circ T d \mu=\lim _{m \rightarrow \infty} \int f \circ \widetilde{P}_{m} g d \mu=\int f g d \mu .
$$

Combining the results from (4) and (5)

$$
\int f d \mu_{T}=\int f g d \mu .
$$

Since $f$ is an arbitrary bounded continuous function with support in $S\left(x_{0}\right)$ by a straightforward approximation it follows that for any measurable set $\Lambda \subset S\left(x_{0}\right)$

$$
\int_{\Lambda} d \mu_{T}=\int_{A} g d \mu
$$

Therefore on $S\left(x_{0}\right)$

$$
\frac{d \mu_{T}}{d \mu}=g
$$

Now,

$$
B=\bigcup_{x \in B} S(x)
$$

and by the separability of $B$ there is a countable dense collection $\left(x_{n}\right)_{n \in N}$ such that

$$
B=\bigcup_{n=1}^{\infty} S\left(x_{n}\right) .
$$

For $n=1$ using uniform integrability there is a subsequence that converges in $\sigma\left(L^{1}, L^{\infty}\right)$ to a function with support in $S\left(x_{1}\right)$. Proceeding inductively for $n$ apply this technique to the subsequence obtained at the $n-1$ step. The diagonal sequence is a subsequence of the original sequence that converges in $\sigma\left(L^{1}, L^{\infty}\right)$ on each $S\left(x_{n}\right) n \in N$. Since it is trivial to verify on overlapping neighborhoods $S\left(x_{i}\right)$ and $S\left(x_{j}\right)$ that the $\sigma\left(L^{1}, L^{\infty}\right)$ limit is consistent, the Radon-Nikodym derivative, $d \mu_{T} / d \mu$, can be constructed inductively on each $S\left(x_{n}\right)$.

For a measurable set $\Gamma \subset B$ define $\Lambda_{1}=S\left(x_{1}\right) \cap \Gamma$ and inductively $\Lambda_{n}=\left(S\left(x_{n}\right) \cap \Gamma\right) \backslash\left(\bigcup_{k=1}^{n-1} \Lambda_{k}\right)$. Thus 


$$
\begin{aligned}
\int_{\Gamma} d \mu_{T} & =\sum_{n=1}^{\infty} \int_{\Lambda_{n}} d \mu_{T} \\
& =\sum_{n=1}^{\infty} \int_{\Lambda_{n}} \frac{d \mu_{T}}{d \mu} d \mu .
\end{aligned}
$$

Therefore, $\mu_{T} \ll \mu$.

ACKNowledgment. The author wishes to thank K. D. Elworthy who made a correction to the original version of the paper.

\section{REFERENCES}

1. R. M. Dudley, J. Feldman and L. LeCam, On seminorms and probabilities, and abstract Wiener spaces, Ann. Math., 93 (1971), 390-408.

2. N. Dunford, and J. T. Schwartz, Linear operators I: General Theory, Interscience New York (1963).

3. L. Gross, Abstract Wiener spaces, Proc. Fifth Berkeley Symp. on Math. Stat. and Prob., University of California Press, 1965.

4. - Measurable functions on Hilbert space, Trans. Amer. Math. Soc., 105 (1962), 372-390.

5. H. H. Kuo, Integration theory on infinite dimensional manifolds, Trans. Amer. Math. Soc., 159 (1971), 57-78.

6. I. E. Segal, Distributions in Hilbert space and canonical systems of operators, Trans. Amer. Math. Soc., 88 (1958), 12-41.

Received March 23, 1973 and in revised form March 8, 1974. Research partially supported by National Science Foundation Grant number GK-32136.

State University of New York, Stony Brook 



\section{PACIFIC JOURNAL OF MATHEMATICS}

EDITORS

RICHARD ARens (Managing Editor)

University of California

Los Angeles, California 90024

\section{R. A. Beaumont}

University of Washington

Seattle. Washington 98105

\section{J. DugundjI}

Department of Mathematics University of Southern California Los Angeles, California 90007

D. Gilbarg and J. Milgram

Stanford University

Stanford. California 94305

\section{ASSOCIATE EDITORS}
E. F. BECKENBACH
B. H. NEUMANN
F. WOLF
K. Yoshida

\section{SUPPORTING INSTITUTIONS}

\author{
UNIVERSITY OF BRITISH COLUMBIA \\ CALIFORNIA INSTITUTE OF TECHNOLOGY \\ UNIVERSITY OF CALIFORNIA \\ MONTANA STATE UNIVERSITY \\ UNIVERSITY OF NEVADA \\ NEW MEXICO STATE UNIVERSITY \\ OREGON STATE UNIVERSITY \\ UNIVERSITY OF OREGON \\ OSAKA UNIVERSITY
}

\author{
UNIVERSITY OF SOUTHERN CALIFORNIA \\ STANFORD UNIVERSITY \\ UNIVERSITY OF TOKYO \\ UNIVERSITY OF UTAH \\ WASHINGTON STATE UNIVERSITY \\ UNIVERSITY OF WASHINGTON

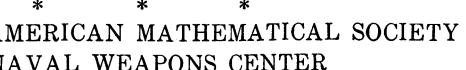

The Supporting Institutions listed above contribute to the cost of publication of this Journal, but they are not owners or publishers and have no responsibility for its content or policies.

Mathematical papers intended for publication in the Pacific Journal of Mathematics should be in typed form or offset-reproduced, (not dittoed), double spaced with large margins. Underline Greek letters in red, German in green, and script in blue. The first paragraph or two must be capable of being used separately as a synopsis of the entire paper. Items of the bibliography should not be cited there unless absolutely necessary, in which case they must be identified by author and Journal, rather than by item number. Manuscripts, in duplicate if possible, may be sent to any one of the four editors. Please classify according to the scheme of Math. Rev. Index to Vol. 39. All other communications to the editors should be addressed to the managing editor, or Elaine Barth, University of California, Los Angeles, California, 90024.

100 reprints are provided free for each article, only if page charges have been substantially paid Additional copies may be obtained at cost in multiples of 50 .

The Pacific of Journal Mathematics is issued monthly as of January 1966. Regular subscription rate: $\$ 72.00$ a year (6 Vols., 12 issues). Special rate: $\$ 36.00$ a year to individual members of supporting institutions.

Subscriptions, orders for back numbers, and changes of address should be sent to Pacific Journal of Mathematics, 103 Highland Boulevard, Berkeley, California, 94708.

\section{PUBLISHED BY PACIFIC JOURNAL OF MATHEMATICS, A NON-PROFIT CORPORATION}

Printed at Kokusai Bunken Insatsusha (International Academic Printing Co., Ltd.), 270, 3-chome Totsuka-cho. Shinjuku-ku, Tokyo 160. Japan.

Copyright (C) 1973 by Pacific Journal of Mathematics Manufactured and first issued in Japan 


\section{Pacific Journal of Mathematics}

\section{Vol. 52, No. $2 \quad$ February, 1974}

Harm Bart, Spectral properties of locally holomorphic vector-valued functions .....

J. Adrian (John) Bondy and Robert Louis Hemminger, Reconstructing infinite

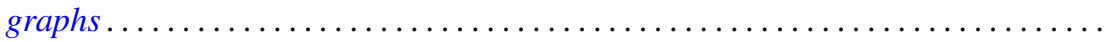

Bryan Edmund Cain and Richard J. Tondra, Biholomorphic approximation of planar

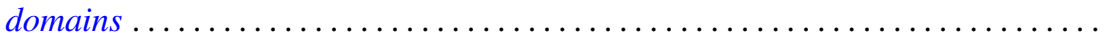

Richard Carey and Joel David Pincus, Eigenvalues of seminormal operators,

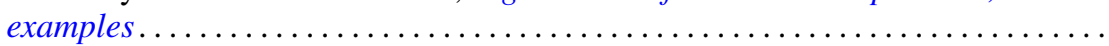

Tyrone Duncan, Absolute continuity for abstract Wiener spaces . . . . . . . . . . . . Joe Wayne Fisher and Louis Halle Rowen, An embedding of semiprime

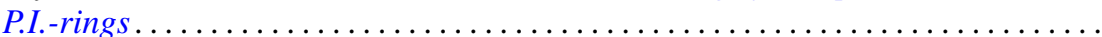

Andrew S. Geue, Precompact and collectively semi-precompact sets of semi-precompact continuous linear operators. . . . . . . . . . . . . . . .

Charles Lemuel Hagopian, Locally homeomorphic $\lambda$ connected plane continua ..... . Darald Joe Hartfiel, A study of convex sets of stochastic matrices induced by

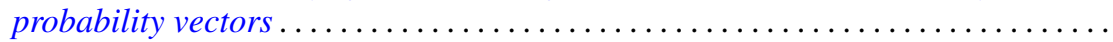

Yasunori Ishibashi, Some remarks on high order derivations $\ldots \ldots \ldots \ldots \ldots \ldots \ldots$ Donald Gordon James, Orthogonal groups of dyadic unimodular quadratic forms.

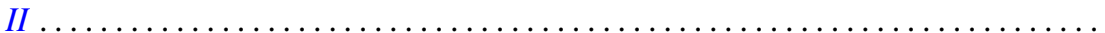

Geoffrey Thomas Jones, Projective pseudo-complemented semilattices . . . . . . . . . Darrell Conley Kent, Kelly Denis McKennon, G. Richardson and M. Schroder,

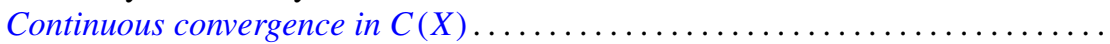

J. J. Koliha, Some convergence theorems in Banach algebras ...

Tsang Hai Kuo, Projections in the spaces of bounded linear oper

George Berry Leeman, Jr., A local estimate for typically real functions . .

475

Andrew Guy Markoe, A characterization of normal analytic spaces by the

homological codimension of the structure sheaf .........

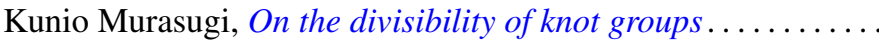

John Phillips, Perturbations of type I von Neumann algebras.

Billy E. Rhoades, Commutants of some quasi-Hausdorff matrices . .

David W. Roeder, Category theory applied to Pontryagin duality

Maxwell Alexander Rosenlicht, The nonminimality of the differential closure .

Peter Michael Rosenthal, On an inversion theorem for the general Mehler-Fock transform pair.

Alan Saleski, Stopping times for Bernoulli automorphisms

John Herman Scheuneman, Fundamental groups of compact complete locally affine complex surfaces. II. ........................

Vashishtha Narayan Singh, Reproducing kernels and operators with a cyclic vector. I. .

Peggy Strait, On the maximum and minimum of partial sums of random variables.

J. L. Brenner, Maximal ideals in the near ring of polynomials modulo 2 .

Ernst Gabor Straus, Remark on the preceding paper: "Ideals in near rings of polynomials over a field" ..........................

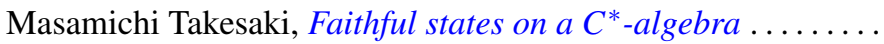

R. Michael Tanner, Some content maximizing properties of the regular simplex.

Andrew Bao-hwa Wang, An analogue of the Paley-Wiener theorem for certain

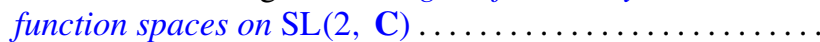

